Objectives: The aims of our study were to assess the 10-year survival of the first TNF-i, to compare retention rates of different anti-TNF drugs in real life settings and to identify factors associated with drug retention in active axSpA.

Methods: We performed a hospital-based retrospective cohort study on consecutive adult axSpA suboptimally controlled by standard therapy, starting their first biological agent with infliximab (IFX), adalimumab (ADA), etanercept (ETA) or golimumab (GLM) according to local policy, recruited at three academic centres between 2003 and July 2018.

Drug efficacy (BASDAI, ASDAS-CRP) as well as reasons for discontinuation were evaluated every 24 weeks. Drug survival was calculated using the Kaplan-Meier analysis, while univariate and multivariate regression was used for predictors of persistence and withdrawal $(p<0.05)$. Subanalysis was done according to discontinuation reasons.

Results: Of the 241 axSpa were recruited, 104 (43.15\%) cases received ETA (original, biosimilar), 100 (41.49\%) ADA, 26 (10.78\%) IFX (original and biosimilar) and 11 cases (4.56\%) GLM.

Statistical significant improvement was demonstrated (ASDAS-CRP, BAS$\mathrm{DAl}, \mathrm{BASFI}$ ) in all patients, those with higher disease activity and functional impairment at baseline presenting earlier and higher response rate $(p<0.05)$.

We reported high long-term persistence of the first TNF-i with a median survival rate of $8.1 \pm 2.1$ years for IFX, ADA and ETA; furthermore, at 10 years, up to one third $(36 \%)$ of axSpA remained on the initial drug achieving either stable remission $(62.24 \%)$ or low disease activity $(37.76 \%)$, while one out of five patients on the same drug after 140 months. The retention rates of ETA, ADA and IFX were $70 \%, 68 \%$ and $57 \%$ after 3 years; $68 \%, 48 \%$ and $53 \%$ after 5 years; $35 \%, 30 \%$ and $27 \%$ after 10 years. Overall, retention to ETA was superior to that of monoclonal antibodies $(p<0.05)$, with a total drug-exposure of 625.43 patient-years for ETN, 415.26 for ADA, 221.53 for IFX.

In addition, survival of the second TNF drug was good but inferior to the first TNF-I $(p<0.05)$

Male sex, age under 40, high baseline $C$ reactive protein, low initial BASFI and disease duration under 5 years were associated with retention rate in multivariate analysis $(p<0.05)$, while the presence of syndesmophytes and obesity with higher withdrawal $(p<0.05)$

Conclusion: We reported high long-term persistence of the first biological agent in axSpA, with superior retention for ETA compared to monoclonal antibodies. Predictors for high retention rate advocate the rationale for the drug choice in different axSpA settings.

Disclosure of Interests: CODRINA ANCUTA Speakers bureau: Abbvie, Pfizer, Novartis, MSD, Roche, Biogen, UCB, Lilly, Cristina Pomirleanu Speakers bureau: Abbvie, Pfizer, UCB, Raluca Paiu: None declared, Georgiana Strugariu Speakers bureau: Abbvie, Pfizer, UCB, Luiza Petrariu Speakers bureau: Abbvie, Pfizer, Novartis, Eugen Ancuta: None declared, Codruta Bran Speakers bureau: Abbvie, Pfizer, Novartis, Lilly, Roche, Rodica Chirieac: None declared, Claudia Mihailov Speakers bureau: Abbvie, Pfizer, Novartis, MSD, Roche, Biogen, UCB, Lilly

DOI: 10.1136/annrheumdis-2019-eular.4272

\section{FRI0383 PAMIDRONATE IN CHRONIC NON-BACTERIAL OSTEOMYELITIS: A RANDOMIZED, PLACEBO- CONTROLLED PILOT TRIAL}

Caroline Marie Andreasen ${ }^{1}$, Anne Grethe Jurik ${ }^{2}$, Bent Deleuran ${ }^{1,3}$, Troels Bille Folkmar ${ }^{4}$, Hans Christian Horn ${ }^{5}$, Troels Herlin ${ }^{6}$, Ellen-Margrethe Hauge ${ }^{1,7}$ ${ }^{1}$ Aarhus University Hospital, Dept. of Rheumatology, Aarhus C, Denmark; ${ }^{2}$ Aarhus University Hospital, Dept. of Radiology, Aarhus C, Denmark; ${ }^{3}$ Aarhus University, Department of Biomedicine, Aarhus, Denmark; ${ }^{4}$ Aarhus University Hospital, Dept. of Oral and Maxillofacial Surgery, Aarhus, Denmark; ${ }^{5}$ Odense University Hospital, Dept. of Rheumatology, Odense, Denmark: ${ }^{6}$ Aarhus University Hospital, Paediatrics, Aarhus, Denmark; ${ }^{7}$ Aarhus University, Dept. of Clinical Medicine, Aarhus, Denmark

Background: Chronic non-bacterial osteitis $(\mathrm{CNO})$ is an autoinflammatory disease often associated with seronegative spondyloarthritides. Osteitic bone lesions in adults are predominantly located in the anterior chest wall, the spine and pelvis. Case series suggest that the amino-bisphosphonate pamidronate may be effective in improving bone inflammation, pain, and disability. No randomized controlled trials have been conducted in CNO.

Objectives: In a randomized double-blinded, placebo-controlled trial we hypothesized that pamidronate reduces radiological signs of bone inflammation assessed by whole-body magnetic resonance imaging, improve pain, disability and reduces systemic markers of bone inflammation and bone turnover in blood.
Methods: Patients with an established diagnosis of CNO were assessed for eligibility. Inclusion criteria were 1. Mono- or multifocal bone inflammation (osteitis with or without sclerosis) 2. Jansson clinical score for CNO in children and adults $\geq 39^{1}$ or malignancy and infection excluded by biopsy and 3 . Symptoms duration $>6$ weeks. Patients were randomized $1: 1$ to receive placebo or pamidronate $1 \mathrm{mg} / \mathrm{kg}$, $\max 60 \mathrm{mg}$, given for three consecutive days at baseline, week 12 and week 24. Whole-body MRI was performed at baseline, week 12 and 36. CT scan of the anterior chest wall was performed at baseline and week 36 . Changes in active and chronic radiological bone inflammation in the ACW and spine were systematically scored. ${ }^{2}, 3$ Patient-reported outcome were measured at baseline and week 1,4,12,24 and 36 using a visual analogue scale for pain and global health assessment, Health Assessment Questionnaire for physical functioning and, EuroQoL- 5 Dimension-3 Level and The Short Form (SF)-36 questionnaire for Quality of life. Biomarkers of bone turnover and inflammation in blood were measured at baseline and week $1,4,12,24$ and 36

Results: Analyses were performed in 12 patients with $\mathrm{CNO}$, mean age 32 (range 20-63) years. From baseline to week 36 the radiological score of active bone inflammation decreased from 4,8(SD 2.2) to 2,5 (SD 1.6) in the pamidronate group $(p=0,01)$. This differed significantly from the placebo group in which no change from baseline was observed $(p=0.04)$ We observed a decreasing trend in VAS pain from mean 38(SD 23)mm to $19(\mathrm{SD} 18) \mathrm{mm}$ and VAS global from $32(\mathrm{SD} 22) \mathrm{mm}$ to $13(\mathrm{SD} 18) \mathrm{mm}$. Conclusion: This study is the first randomized double-blinded, placebo controlled trial in CNO. Treatment with pamidronate improved bone marrow oedema in the anterior chest wall assessed by whole-body magnetic resonance imaging. This pilot study points out methodological considerations in relation to study design and outcome measures in CNO.

\section{REFERENCES:}

[1] Jansson AF, Müller TH, Gliera L, Ankerst DP, Wintergerst U, Belohradsky $\mathrm{BH}$, et al. Clinical score for nonbacterial osteitis in children and adults. Arthritis Rheum 2009:60:1152-9.

[2] Weber U, et al. Anterior chest wall inflammation by whole-body magnetic resonance imaging in patients with spondyloarthritis: lack of association between clinical and imaging findings in a cross-sectional study. Arthritis Res Ther 2012;14:R3.

[3] Madsen KB, Jurik AG. MRI grading method for active and chronic spinal changes in spondyloarthritis. Clin Radiol 2010;65:6-14.

Disclosure of Interests: None declared

DOI: 10.1136/annrheumdis-2019-eular.7139

\section{FRI0384 IMPACT OF INTERLEUKIN 17 BLOCKING AGENT ON CLINICAL OUTCOME IN SAPHO PATIENTS}

Gunter Assmann ${ }^{1}$, Joerg, Thomas Bittenbring ${ }^{1}$, Annette D. Wagner ${ }^{2}$,

Martin Schreiber ${ }^{3}$, Konstantinos Christofyllakis ${ }^{1}$, Ulrike Hueffmeier ${ }^{4}$,

Claudia Pfoehler ${ }^{5}$, Frank Neumann ${ }^{6} .{ }^{1}$ University Medical School of Saarland,

Medicine I, Oncology and Rheumatology, Homburg, Germany, ${ }^{2}$ University School of Medicine Hannover, Department of Nephrology, Hannover, Germany; ${ }^{3}$ Hospital of Worms, Department of Radiology, Worms, Germany; ${ }^{4}$ University Medical School of Erlangen, Human Genetics, Erlangen, Germany; ${ }^{5}$ University Medical School of Saarland, Department of Dermatology, Homburg, Germany; ${ }^{6}$ University Saarland Medical School, Jose Carreras Center for Immunogenetics, Homburg, Germany

Background: SAPHO syndrome has to be considered as a rare subtype of seronegative spondyloarthritis (SpA) showing typical manifestations with palmoplantar pustolosis and osteitis with hyperostosis; the clinica response of conventional or biological disease modifying drugs (DMARDs) in SAPHO syndrome are often disappointing. Whereas in SpA peripheral arthritis and inflammatory back pain represent the leading symptoms, the SAPHO patients often complain painful osteitis with hyperostosis in the sternal region as well as the palmoplantar pustulosis accompanied by synovitis preferentially in large joints including sacroiliitis. Recently, the detection of higher numbers of CD4+IL17+ lymphocytes in the peripheral blood of SAPHO patients has arisen the hypothesis that Th4/17 helper cells with their secretion of interleukin 17 (IL17) could be involved in the development of inflammation in SAPHO syndrome (1)

Objectives: Here we present an observational study of 12 SAPHO patients which were treated with the IL 17 blocking agent secukinumab. In addition, the fraction of CD4/17+ lymphocytes in peripheral blood specimen has been monitored on treatment.

Methods: Between January 2015 and February 2017 clinical activity of disease were measured in 37 SAPHO patients with a disease duration of 11 years (median). The disease activity were evaluated by the osteitis 